\title{
Organizaciones sociales de pobladores y prácticas de resistencia en contexto de pandemia por COVID-19 en Chile
}

\author{
Patricia Castañeda-Meneses \\ Doctora en Ciencias de la Educación. Trabajadora social \\ Universidad de Valparaíso. Valparaíso, Chile \\ https://orcid.org/0000-0002-4676-5872・patricia.castaneda@uv.cl
}

Resumen

El artículo presenta los resultados de una investigación que tiene como objetivo caracterizar el funcionamiento de las ollas comunes de las comunas de Valparaíso y Viña del Mar, Chile, como prácticas de resistencia implementadas por las organizaciones sociales de pobladores durante la actual crisis sanitaria por COVID-19. Metodológicamente se desarrolla una investigación cualitativa de carácter descriptivo en cuatro organizaciones sociales, a partir de secuencias de observación no participante y revisión documental de sus publicaciones en redes sociales, validando la información por triangulación temporal y de técnicas. Los resultados obtenidos constatan que las actuales organizaciones actualizan una práctica histórica, impulsada por el incremento de la escolaridad formal y por el manejo tecnológico de las nuevas generaciones. Las prácticas de resistencia se expresan a través de tres dimensiones: denuncia de la precariedad económica de las familias que impide satisfacer sus necesidades de alimentación en forma autónoma; desafío al modelo económico vigente, buscando soluciones colectivas por sobre el individualismo y la competitividad; y dignidad, acogiendo en sus actividades a la diversidad de sujetos sociales presentes en el territorio. Se concluye que la acción transformadora de estas prácticas se realiza a partir de redes colaborativas y de apoyo entre pares, visibilizando importantes consecuencias políticas desde su quehacer, al revelar las tramas del tejido social que enfrentan la pandemia con un nivel de precariedad y carencia de tal profundidad, que los aportes provenientes de recursos públicos y de las acciones de beneficencia no aportan coberturas mínimas de satisfacción de necesidades básicas.

Palabras clave: Prácticas de resistencia; Organizaciones sociales de pobladores chilenas; Necesidades básicas; Emergencia sanitaria por COVID-19.

Recibido: 23/08/2021 | Aprobado: 19/10/2021 | Publicado: 01/01/2022

(1) (2) Esta obra está bajo una Licencia Creative Commons Atribución-NoComercialCompartirIgual 4.0 Internacional.

Financiación o proveniencia del artículo: Artículo derivado de proyecto de investigación independiente adscrito a las actividades académicas de la Escuela de Trabajo Social de la Universidad de Valparaíso, Chile, desarrollado en el periodo 2020-2021.

¿Cómo citar este artículo? / How to quote this article?

Castañeda-Meneses, P. (2022). Organizaciones sociales de pobladores y prácticas de resistencia en contexto de pandemia COVID-19 en Chile. Prospectiva. Revista de Trabajo Social e intervención social, (33), 13-32. doi: 10.25100/prts.v0i33.11541. 
Castañeda-Meneses

\title{
Social Organizations of People and Practices of Resistance in Times of COVID-19 in Chile
}

\begin{abstract}
The article presents the results of an investigation that aims to characterize the functioning of the community kitchens of the communes of Valparaíso and Viña del Mar, Chile as resistance practices implemented by social organizations of residents during the current health crisis of COVID-19. Methodologically, qualitative-descriptive research was carried out in four social organizations, based on non-participating observation sequences and documentary review of publications on social networks, validating information by temporal triangulation and techniques. The results show that the current organizations update a historical practice, driven by the increase in formal schooling and by the management of digital technology by the new generations. The practices of resistance are expressed in three dimensions: denunciation of the economic precariousness of families that prevents them from meeting their nutrition needs autonomously; challenge to the current economic model, seeking collective solutions over individualism and competitiveness; and dignity, welcoming in their activities the diversity of social subjects present in the territory. It is concluded that the transformative action of these practices is carried out from collaborative networks and peer support, highlighting important political consequences.

Keywords: Practices of resistance; Social organizations of people; Basic needs, Health emergency of COVID-19.
\end{abstract}

Sumario: 1. Introducción, 2. Metodología, 3 Hallazgos, 4. Conclusiones. 


\section{Introducción}

A partir del 18 de octubre del 2019, Chile enfrentó un grave episodio de crisis social que se caracterizó por masivas manifestaciones a lo largo del territorio nacional y que expresaban un abierto malestar social acompañado de profundos cuestionamientos a la institucionalidad económica y política vigente en el país, basada en el modelo de libre mercado y en la implementación del rol estatal subsidiario. Esta institucionalidad heredada desde tiempos dictatoriales ha generado durante sus cinco décadas de vigencia profundas desigualdades en el acceso a los beneficios del desarrollo nacional, ocasionando una alta concentración del ingreso, importantes rangos de endeudamiento de los sectores de ingresos medios y bajos, privatización de los servicios sociales y traspaso de las lógicas mercantilistas a las políticas sociales, traducidas en procesos de medición tecnocrática de la pobreza y en la asignación focalizada de recursos escasos (Morales-Quiroga, 2020). Consecutivamente, en marzo del 2020 y sin alcanzar a decantarse plenamente la crisis social anterior, se superpone un segundo escenario asociado a una crisis sanitaria global, formalizada a partir de la declaración oficial de pandemia por COVID-19 que realiza la Organización Mundial de la Salud y que ha obligado a todos los Estados a enfrentar esta emergencia con medidas de prevención restrictivas de la movilidad individual (Ramos, 2020). Lo anterior ha afectado negativamente el funcionamiento regular de las actividades económicas, sociales y culturales en las diversas regiones del mundo y ha obligado a poner en marcha un conjunto de acciones asistenciales y paliativas que buscan proteger la salud, el empleo y las condiciones de calidad de vida de la población en situaciones de confinamiento forzado. En consecuencia, el Gobierno chileno a través del Ministerio de Salud ha implementado una serie de medidas sanitarias que buscan proteger a la población del riesgo de contagio, junto con gestionar los recursos, la cobertura y la oportunidad de atención especializada requerida para enfrentar la pandemia (Ministerio de Salud de Chile [MINSAL], 2020). Simultáneamente, ha impulsado a través de los Ministerios de Hacienda, Economía, Desarrollo Social y Familia, una serie de medidas económicas paliativas en el marco de las reglas subsidiarias vigentes, dirigidas a sectores sociales focalizados que se han visto afectados en sus ingresos familiares debido a las sucesivas crisis acontecidas en el país y a las restricciones en la movilidad personal que representa la emergencia sanitaria. Estas medidas corresponden a subsidios monetarios, créditos blandos y aportes en mercaderías e insumos básicos, los que se han asignado en secuencias temporales parciales de meses predefinidos $\mathrm{y}$ han resultado insuficientes para resolver las necesidades básicas permanentes asociadas a la subsistencia (Gobierno de Chile, 2020). Lo anterior ha impulsado a las organizaciones sociales de pobladores a implementar una serie de acciones que buscan enfrentar colectivamente situaciones críticas asociadas a la satisfacción de necesidades básicas, destacando entre estos esfuerzos la actualización de las iniciativas históricas denominadas ollas comunes, como alternativas implementadas para enfrentar la necesidad de alimentación de la población desde lógicas de organización solidaria (Guerrero-Jiménez y Pérez-Mora, 2020). 
Estas acciones solidarias encuentran su génesis histórica en el país en las organizaciones sociales de pobladores, las que representan manifestaciones de acciones colectivas realizadas a lo largo de las décadas que han permitido su constitución como sujeto social en la sociedad chilena, con presencia y reconocimiento público (Valdés, Weinstein y Malinarich, 1988). En el marco de esta dinámica social, Herrera (2018) identifica dos líneas distintivas de las organizaciones sociales de pobladores. La primera línea corresponde a las organizaciones sociales vinculadas a la reivindicación por el acceso a la vivienda, las que comienzan su accionar a mediados del siglo XX a través de la ocupación de hecho de predios urbanos, expresiones colectivas conocidas como "tomas de terrenos" y que han dado origen a procesos de autoconstrucción y regularización de la propiedad habitacional. Esta organización poblacional con fines reivindicativos ha permitido el acceso forzado a soluciones habitacionales, infraestructura comunitaria y servicios básicos a amplios sectores de la población en situación de pobreza, especialmente en zonas urbanas y periurbanas. La segunda línea corresponde a la organización social orientada a la supervivencia, la satisfacción de las necesidades básicas y la defensa de los Derechos Humanos. En estos casos, la organización es una expresión de protesta social, que se manifiesta con especial atención en períodos de crisis económicas o de acción represiva estatal, destacando en este marco la acción poblacional realizada durante las décadas dictatoriales de 1970 y 1980 (Iglesias-Vázquez, 2016).

Para Razeto-Migliaro (1986), el análisis específico de la experiencia realizada en la dictadura por parte de las organizaciones de pobladores, orientadas a la protesta social, demostró que facilitaron la promoción de la participación democrática, autogestionada y autónoma en sectores populares, expresada en la generación y mantención de relaciones solidarias, de cooperación y ayuda mutua, las que permitieron redefinir a la comunidad como un territorio de autoafirmación y de resistencia. La acción organizacional reivindicativa se desplegó a través de redes de apoyo integrales, las que actuaron en respuesta a las insuficiencias de la institucionalidad pública para satisfacer las necesidades básicas de la población. Esta situación de insuficiencia se caracterizaba por estar acompañada de acciones represivas ejercidas por agentes de seguridad de la dictadura, las que mostraron especial encono con los sectores poblacionales, sindicales, campesinos, estudiantiles y universitarios del país; y reflejaba los devastadores efectos económicos generados por la forzada reorientación del Estado desde un rol protector a un rol subsidiario, cambio estructural impulsado unilateralmente a partir de la década de 1970 por los grupos de interés a cargo de la economía del país en esa época. Estas redefiniciones fueron acompañadas por la eliminación de las medidas de protección de la producción fabril nacional con el propósito de abrir la economía a las reglas del libre mercado, lo que ocasionó la quiebra de parte importante de la industria tradicional, la que a esa fecha constituía la principal empleadora de amplios grupos poblacionales urbanos, quienes vieron afectados sus ingresos familiares por la cesantía y el subempleo (Ffrench-Davis y Stallings, 2001). En ese contexto, las organizaciones sociales de pobladores contribuyeron a la reconstrucción 
del tejido social profundamente rasgado por la represión política, permitiendo el reordenamiento de las fuerzas sociales y políticas en acciones de corto plazo expresadas en resistencia territorial y protesta social contra la dictadura; y en el mediano plazo, acompañando el dificultoso trazado de retorno a la democracia por el que debió transitar el país a finales de la década de 1980 e inicios de los años 90 (Berlagoscky, Infante y De Ferrari, 2018). Sin embargo, puede afirmarse que esta histórica capacidad de organización de los sectores poblacionales frente a las situaciones de crisis social fue desconocida por las políticas postdictatoriales de superación de la pobreza, las que definieron a las familias pobladoras desde un rol beneficiario receptor pasivo, sin reconocerlas como agentes sociales y políticos activos (Angelcos, 2016).

Las iniciativas de las organizaciones de pobladores son prácticas sociales que se caracterizan por conformar "un conjunto de acciones producidas por agentes sociales concretos en situaciones significativas para ellos" (Jociles-Rubio, 2018, p 122). En específico, las prácticas sociales se transforman en prácticas de resistencia al definir una posición política basada en la comunidad, que busca hacer posible una acción transformadora que valora lo relacional y la interdependencia por sobre la acción individual (Núñez-Puente, Fernández-Romero y Farné, 2018). Su justificación, sus antecedentes y sus consecuencias como práctica se encuentran enmarcados en lugares, espacios y tiempos específicos, que las relacionan con un marco cultural que las define, les otorga sentidos distintivos y las proyecta a un mundo social de mayor amplitud, en donde aportan contrapuntos críticos respecto de los pensamientos y acciones sociales hegemónicas (Langer, 2016). Para Collignon-Goribar (2010) una práctica social posee atributos de resistencia cuando se enfrenta abiertamente a los referentes tradicionales que regulan dicha práctica y que se imponen por uso o costumbre como criterios únicos para la toma de decisiones. Como resultado se produce una tensión social que expresa la presencia de posiciones antagónicas que por una parte, presionan por la mantención del orden establecido y por otra, buscan provocar un cambio social que modifique parcial o totalmente los parámetros vigentes al momento de suceder el enfrentamiento.

Específicamente, las prácticas de resistencia pueden ser conceptualizadas como “todas aquellas formas de acción, sean colectivas o individuales, ejercidas por miembros de una clase subordinada, que limitan la extracción de excedentes o que son capaces de afectar la intensidad con la cual el excedente es extraído" (Piñeiro, 1985, p. 24). Complementariamente, Jarpa-Arriagada (2020), plantea que las prácticas de resistencia comparten los atributos de cuestionamiento al capitalismo y al modelo de desarrollo neoliberal, expresándose en una clara oposición a las relaciones de dominación que afectan a amplios grupos sociales. Por su parte, Barreneche, Lombada-Bermúdez Ramos-Martín (2021) identifican en la base de las resistencias, el rechazo social al control normativo que ejerce la tecnocracia y la dominación tecnológica en la implementación de las políticas sociales; mientras que Alves da Silva y Barreto-Silva (2020) las describen como una 
diversidad de iniciativas de reconocimiento que enfrentan la exclusión social en base a estrategias colectivas que buscan reducir la subordinación y explotación generada en el modelo económico vigente y acceder a políticas públicas que garanticen derechos sociales.

En el actual contexto nacional, las prácticas políticas y económicas predominantes se encuentran asociadas a procesos de acumulación y apropiación, en donde la satisfacción de las necesidades básicas es resuelta preferentemente por las lógicas de funcionamiento del mercado, a través de mecanismos de oferta de productos y servicios destinados al consumo masivo, acompañado del acceso abierto al crédito y al endeudamiento de las personas y las empresas. La emergencia de prácticas de resistencia es un contrapunto que se expresa por medio de iniciativas comunitarias que buscan rescatar la cooperación, la reciprocidad y la autogestión por sobre las lógicas mercantiles vigentes en el país, concibiendo las necesidades sociales como demandas o reivindicaciones expresadas por sujetos sociales o territorios definidos, que representan acciones de afirmación y resistencia orientadas a rescatar y valorizar estrategias colectivas de movilización social o incidencia en los procesos de toma de decisiones, los que actualmente asumen preferentemente la condición de transacciones entre grupos de elite pertenecientes a las esferas políticas y económicas (Alves da Silva y Barreto-Silva, 2020).

Desde la perspectiva de las organizaciones sociales, las ollas comunes constituyen prácticas de resistencia que representan una respuesta colectiva frente a la necesidad básica de alimentación y se sustentan en el trabajo colaborativo y autogestionado. Gallardo (1985a, 1985b) las concibe como iniciativas que han estado presentes a lo largo de la historia nacional, reconociendo sus principales hitos a partir de las huelgas obreras salitreras de inicios del siglo XX; en las acciones caritativas permanentes de la iglesia católica y de grupos voluntarios realizadas a lo largo de los años en sectores en situación de pobreza; como acciones complementarias a los procesos de ocupaciones de terrenos sucedidos en las décadas de 1950 y 1960; y como manifestación de la carencia material y la represión política ejercida durante la dictadura cívico militar iniciada en septiembre de 1973. Específicamente en lo que respecta al funcionamiento de las ollas comunes durante los años dictatoriales, Hardy (1986) las reconoce como un histórico instrumento de denuncia social, que constituyó una respuesta al marco de exclusión económica, social y política que afectó a vastos sectores de la población nacional; caracterizadas a partir de "su carácter de organización popular, con asiento territorial y cuyo objetivo de subsistencia descansa en recursos y trabajo humano aportados colectivamente" (Hardy, 1986, pp. 25-26). Complementariamente, Valdés (1986) reconoce la contribución de las ollas comunes en la reconstitución del tejido social dramáticamente arrasado por la represión política durante el período dictatorial, y cuyas acciones destinadas a la satisfacción de necesidades básicas se erigieron simbólicamente en las principales prácticas de resistencia de los sectores populares de la época y se proyectaron como un importante aporte de organización social en la lucha por el retorno a la democracia. 
En el marco de la actual crisis sanitaria por COVID-19, se ha actualizado el interés de la investigación social por las ollas comunes como espacios de organización de pobladores que actualizan las prácticas de resistencias históricas que les antecedieron. Los estudios realizados a la fecha han centrado su interés en el aporte que realizan las mujeres en el desarrollo de estas iniciativas, permitiendo sumar la perspectiva de género en el análisis del contexto de crisis sanitaria (Palacios-Sepúlveda, 2020; Valenzuela-Contreras, 2021). Asimismo, las prácticas de resistencia a través de ollas comunes se reconocen como una expresión social que ha concadenado la crisis social del año 2019 con la crisis sanitaria iniciada el 2020 (Guerrero-Jiménez y Pérez-Mora, 2020). No obstante, dado que los procesos de investigación en torno a esta temática se están realizando en forma sincrónica con las diversas expresiones que adopta la organización social frente a la necesidad de alimentación en pandemia, no se cuenta a la fecha con una información plenamente consolidada a este respecto, por lo que todo esfuerzo en este sentido es concebido como un aporte a la comprensión de un fenómeno social que se encuentra en abierta configuración.

Es por ello que la presente investigación busca contribuir en este sentido, planteándose como objetivo caracterizar el funcionamiento de las ollas comunes de las comunas de Valparaíso y Viña del Mar, Región de Valparaíso, Chile; en su condición de prácticas de resistencia implementadas por las organizaciones sociales de pobladores durante la actual crisis sanitaria por COVID-19. En este marco, y tomando como referentes los fundamentos histórico sociales revisados en el presente apartado y el contexto del escenario de crisis social y sanitaria que atraviesa el país actualmente, se busca contribuir al esclarecimiento de las reelaboraciones de las prácticas de resistencia en los contextos contemporáneos, para contribuir a identificar los desafíos que estos procesos sociales representan para las acciones profesionales de Trabajo Social, especialmente frente a su imperativo ético de acoger, colaborar y visibilizar las acciones solidarias demandadas por la emergencia sanitaria, dado el profundo impacto que representan en la sobrevivencia de los sectores sociales de menores ingresos.

\section{Metodología}

La presente investigación se realizó desde un enfoque cualitativo y posee un carácter descriptivo, permitiendo caracterizar el contexto, las interacciones y las situaciones de vida en que los sujetos desarrollan sus prácticas de resistencia en las organizaciones sociales de pobladores que funcionan en las comunas seleccionadas (Valles, 2000). La unidad de análisis corresponde a organizaciones de pobladores que se ubican en las comunas de Valparaíso y Viña del Mar, Región de Valparaíso, Chile. Las comunas fueron seleccionadas a partir de la alta concentración de población regional que poseen, la que alcanza al 35\% del total regional (Instituto Nacional de Estadísticas Chile [INE], 2017), cifra que respalda una importante dinámica organizacional con fines de representatividad territorial, habitacional, económica, deportiva, recreativa, artística, cultural y de expresión de necesidades de sujetos sociales 
específicos como población mayor, mujeres y jóvenes. Lo anterior dota de amplias posibilidades de identificación y selección de organizaciones que cumplan con los requerimientos declarados para la unidad de análisis. Los criterios de inclusión de las organizaciones sociales correspondieron a contar con una trayectoria que alcanzara al menos cinco años de funcionamiento regular a la fecha del estudio, que dotara a sus integrantes de una experiencia organizacional relevante en la gestión de acciones solidarias en torno a las necesidades de su población, sector o territorio; que hubiese sostenido el funcionamiento ocasional o estable de una olla común durante el último año; y finalmente, las garantías éticas otorgadas a sus dirigentes o responsables de la actividad para declarar abierta y voluntariamente una disposición favorable al proceso de levantamiento de información definido en el marco de la investigación. Los criterios permitieron la selección de dos organizaciones sociales de la comuna de Viña del Mar y dos organizaciones sociales de la comuna de Valparaíso que cumplían con los criterios definidos, permitiendo contar con un total de cuatro contextos organizacionales relevantes para el desarrollo de la investigación. Dado que la investigación se realizó en el marco de cuarentenas obligatorias y restricciones de movilidad que limitaron forzosamente las interacciones entre personas, la información fue recopilada a partir de secuencias de observación no participante, técnica de levantamiento de información que tiene como objeto de estudio describir hechos, objetos o fenómenos de la realidad social, los que recoge en una temporalidad y un escenario específico desde un rol pasivo de la persona investigadora, quien no interviene bajo ninguna circunstancia en el curso de los acontecimientos (Campos y Covarrubias y Lule-Martínez, 2012). Las secuencias de observación no participante fueron realizadas entre los meses de octubre del 2020 y marzo del 2021 en las cuatro organizaciones sociales de pobladores seleccionadas, aportando registros descriptivos del funcionamiento en un día hábil de la semana y un día festivo semanal, nacional o religioso, validando la información recopilada a partir de la triangulación temporal. La información recopilada fue sistematizada a través de transcripciones de las rutinas cotidianas asociadas al funcionamiento de la olla común, a partir de plantillas organizadas según la secuencia horaria y que identificaban los siguientes referentes de observación: condiciones de inicio de la actividad, delegación de tareas, cumplimiento de tareas, preparación de alimentos, modalidades de entrega de la alimentación a las personas beneficiadas, difusión de actividades y organización del próximo día de funcionamiento. Cada registro fue identificado con una sigla distintiva para cada organización $(\mathrm{O} 1, \mathrm{O} 2, \mathrm{O} 3$ y O4) junto con la declaración de la fecha de aplicación de la observación, separada por una diagonal $(\mathrm{O} 1 / \mathrm{dd} / \mathrm{mm} / \mathrm{aa})$. Los códigos resultantes son utilizados como evidencias de respaldo para los resultados de la investigación. Asimismo, se realizó revisión documental de los contenidos de las redes sociales publicados por las organizaciones estudiadas, considerando la influencia de los contenidos en la interacción social y en los esquemas de funcionamiento de la organización social (Rivera-Aguilera, 2017). El uso combinado de las técnicas de observación no participante y revisión documental permitió enriquecer la validación de la información desde el criterio de triangulación de técnicas. Con fines de descripción y análisis de datos, cada revisión fue 
registrada con una sigla distintiva para cada red social y organización (RSO1, RSO2, RSO3 y RSO4) junto con la declaración de la fecha de aplicación de la revisión, separada por una diagonal (RSO1/dd/mm/aa). La información fue organizada reconstruyendo la dinámica cotidiana de las ollas comunes y analizada convergentemente desde el concepto de práctica de resistencia y desde el contexto de emergencia sanitaria por COVID-19. La información recopilada fue analizada a partir de categorías inferenciales válidas y reconocibles para los contextos organizacionales estudiados. Para ello, se realizó identificación y organización de los contenidos en unidades relevantes, permitiendo ilustrar en cada categoría la dinámica cotidiana del funcionamiento de la organización en torno a la entrega de la alimentación a la población beneficiaria. Las consideraciones éticas aplicadas en la investigación consideraron la confidencialidad en el levantamiento y análisis de los datos, y garantías de voluntariedad de la participación y de retiro del proceso en el momento en que se considere apropiado, sin introducir presiones o condicionalidades en la libre participación.

\section{Hallazgos}

En el actual contexto de pandemia y frente a la creciente ausencia de recursos para enfrentar la satisfacción de necesidades básicas a nivel familiar, las organizaciones de pobladores implementan ollas comunes, las que a partir de la información recopilada pueden ser descritas en su funcionamiento desde las siguientes características:

\section{Funcionamiento organizacional horizontal}

A partir de la información recopilada en las secuencias de observación no participantes realizadas, puede constatarse que las tareas iniciales de organización y convocatoria de las ollas comunes a nivel operativo son asumidas por los liderazgos formales de dirigentes sociales o los liderazgos naturales reconocidos a nivel vecinal, quienes asumen roles de facilitación del proceso (O2/08/10/2020; O3/06/10/2021). Esta dinámica refleja una horizontalidad entre integrantes, que tiene sus fundamentos en la ausencia de estructura formal de cargos en la organización y que está orientada por la voluntariedad y el bien común, permitiendo a sus integrantes cumplir con múltiples roles, conforme sean las necesidades de funcionamiento.

Tomando como base la infraestructura, los servicios básicos, el mobiliario y el menaje disponible en los espacios comunitarios, las organizaciones proponen estilos de funcionamiento que les permitan enfrentar sus objetivos, las que por lo general corresponden a comisiones de trabajo específicas orientadas a la manipulación, preparación y entrega de alimentos; aseguramiento del abastecimiento de provisiones e insumos básicos de combustible, higiene y seguridad a través de la recolección de donaciones y aportes; y definición de los sistemas de turnos voluntarios para la preparación de alimentos, atención de la población y mantención de higiene y seguridad de los recintos (O1/09/10/2020, 
Castañeda-Meneses

O2/08/10/2020, O3/06/10/2020 y O4/13/10/2020). Esta dinámica de funcionamiento es marcadamente horizontal, asumiendo sus integrantes una multiplicidad de roles y tareas asociadas a asegurar la preparación y la entrega de alimentos a las personas y familias que lo requieran, junto con enfrentar la responsabilidad en forma conjunta respecto de los procesos de toma de decisiones cotidianas en torno a minuta, número de raciones, compra de mercaderías pendientes, convocatorias y otros aspectos operativos (O3/06/10/2020, O4/21/10/2020, RSO2/14/12/2020). La frecuencia de funcionamiento se define conforme las necesidades de la población y la disponibilidad de recursos diarios y semanales (O1/13/11/2020, RSO4/16/11/2020). La dinámica de funcionamiento se apoya en los recursos tecnológicos provistos a través de videollamadas, información a través de redes sociales y aplicaciones de mensajerías de textos e imágenes (RSO1/22/10/2020; RSO2/18/10/2021; RSO3/07/11/2020 y RSO4/09/11/2020).

\section{Funcionamiento organizacional femenino}

La observación de las secuencias de actividades arroja como resultado que los sistemas de turnos y la conformación de comisiones de trabajo están constituidos preferentemente por mujeres, ya sean dirigentes o madres de familia, las que colaboran en la tarea de preparación de los alimentos como una retribución a la ayuda que reciben cotidianamente (O1/09/10/2020, O2/08/10/2020, O3/06/10/2020 y O4/13/10/2020). En este contexto, su rol tradicional familiar a cargo del cuidado y crianza de hijos e hijas trasciende del espacio privado al espacio público, constituyendo un nuevo espacio colectivo de encuentro entre pares que permite compartir saberes femeninos en torno a la alimentación, los que se encuentran en la base del proceso de elaboración de alimentos que deben realizar diariamente, considerando la disponibilidad de recursos y el número de comensales en cada jornada. Dado que para muchas familias las raciones recibidas constituyen la alimentación principal del día, las mujeres realizan esfuerzos por preparar alimentos de alto aporte nutricional y que garanticen largos períodos de saciedad, por lo que se priorizan preparaciones calientes como legumbres, sopas y guisos, según disponibilidad de los productos (O1/09/10/2020, O2/08/10/2020, O3/06/10/2020 y O4/13/10/2020).

\section{Funcionamiento organizacional autogestionado}

Desde las rutinas registradas, se observa que las actuales condiciones de pandemia reconocidas formalmente en las declaraciones de emergencia sanitaria por COVID-19, han permitido que las organizaciones sociales demanden a diversos organismos públicos y municipios concurran a apoyar estas iniciativas, principalmente a través de alimentos no perecibles (O1/20/11/2020, O4/13/10/2020, RSO1/18/11/2020). Asimismo, se realizan gestiones desde las organizaciones para complementar estas ayudas a través de donaciones recolectadas en ferias hortofrutícolas y en el pequeño comercio, quienes aportan productos 
frescos que mejoran la minuta diaria (O3/30/11/2020, 04/13/10/2021). Complementariamente, se reciben aportes desde establecimientos educacionales, iglesias, empresas, grupos voluntarios o particulares, los que en forma estable o esporádica contribuyen en el funcionamiento de las ollas comunes y comedores solidarios, con especial atención a las fechas de celebraciones nacionales o festividades religiosas, en donde la secuencia de actividades de preparación de alimentos es homóloga a los días de semana, pero se distinguen los esfuerzos realizados para entregar una minuta mejorada por medio de un mayor número de preparaciones disponibles o por la inclusión de alimentos de mayor deseabilidad social, como alimentos de origen animal, aderezos y postres (O1/08/12/2020, O2/24/12/2020).

\section{Funcionamiento organizacional en pandemia}

En el actual marco de prevención de contagios por COVID-19, se observó en forma permanente la entrega de los alimentos directamente en la vajilla de las propias personas beneficiarias, las que son trasladadas a sus hogares para ser consumidos, buscando garantizar con ello el cumplimiento del distanciamiento físico y del confinamiento decretado por las autoridades sanitarias (O1/09/10/2020, O2/08/10/2020, O3/06/10/2020 y O4/13/10/2020). Por ello, no es frecuente observar las tradicionales imágenes de pobladores compartiendo sus alimentos en las mesas de las sedes sociales, aunque la situación puede presentarse en condiciones de excepcionalidad. Asimismo, se constató como particularidades la presencia de familias migrantes, personas mayores y menores de edad, como perfiles priorizados en la entrega de alimentos, junto con el uso universal de las mascarillas y la disponibilidad de alcohol gel en los accesos de las sedes, en su calidad de elementos protectores entre contactos interpersonales (O1/13/11/2020, O2/27/11/2020, O3/30/11/2020 y O4/07/11/2020).

\section{Funcionamiento organizacional en transgresión de normativas vigentes}

En el período de observación realizado, se verificó que a pesar de la declaración formal por parte de las autoridades respecto a restricciones de movilidad por cuarentenas obligatorias con fines de protección sanitaria que buscaban disminuir los riesgos potenciales de contagio, estas medidas no han detenido el funcionamiento regular de la olla común. Por el contrario, las iniciativas siguen funcionando en forma cotidiana y enfrentándose proactivamente a la potencial censura o multa por desafiar las normativas sanitarias. No obstante, pudo pesquisarse que en ocasiones se busca relativizar el cumplimiento de algunas de las restricciones de movilidad por medio del establecimiento de rutas de circulación alternativas en el marco de los pasajes y calles secundarias del sector que realizan las mismas personas asistentes a la olla común y las personas voluntarias que entregan alimentos en domicilio; o definiendo horarios de asistencia en rangos de bajo control externo $(\mathrm{O} 1 / 22 / 03 / 2021, \quad \mathrm{O} 2 / 23 / 03 / 2021, \mathrm{O} 3 / 13 / 03 / 2021, \quad \mathrm{O} 4 / 24 / 03 / 2021$, 
Castañeda-Meneses

RSO1/08/10/2020, RSO3/05/01/2021). Esta postura refleja la concepción de la necesidad de alimentación como emergencia sanitaria mayor a la propia pandemia, y ante la ausencia de un apoyo gubernamental universal las organizaciones sociales continúan funcionando para responder a las necesidades de subsistencia de las familias, que carecen de otros medios para poder afrontarlas. En condiciones de restricción de movilidad para quienes asisten a la olla común, la circulación de información estratégica se realiza a través de la publicación de los horarios de atención y las minutas diarias en aplicaciones de mensajerías de textos e imágenes (RSO2/14/03/2021 y RSO3/12/03/2020).

Funcionamiento organizacional en apoyo a necesidades básicas complementarias a alimentación

La permanente relación de las organizaciones con las familias en situación de precariedad económica, observada a lo largo del estudio, permitió identificar la especial atención que se realiza a las personas afectadas por contagio COVID-19 en sus situaciones desencadenantes de hospitalizaciones, cuarentenas obligatorias de contactos estrechos o confinamiento forzado; ayudas materiales en mercaderías, recursos económicos frescos para enfrentar diversas necesidades familiares y gestión en redes de apoyo para acciones de rehabilitación, trámites en instituciones de salud o celebración de exequias (O1/08/12/2020, O2/27/11/2021 y O4/24/03/2021).

Funcionamiento organizacional en apoyo a los requerimientos tecnológicos de la población

La información recopilada pesquisó que las organizaciones complementan sus acciones asistenciales, diseñando apoyos para la población escolar que realiza sus estudios en modalidad remota (O1/22/03/2021) y para la población mayor que debe desarrollar trámites online para la obtención de autorizaciones de desplazamientos según restricciones de movilidad vigentes y algunas otras gestiones que han sido transferidas desde modalidades presenciales a modalidades virtuales, tales como postulación a beneficios, obtención de certificados, trámites bancarios y similares (O1/22/03/2021y O3/24/10/2020). De manera general, los costos de servicios digitales, nivel de actualización de equipos o teléfonos móviles, acceso a internet y nivel de alfabetización digital de la población usuaria representan dificultades que son acogidas por las organizaciones y acompañadas en su resolución en la medida de la disponibilidad de recursos y apoyos voluntarios (O3/30/11/2020).

\section{Funcionamiento organizacional en redes sociales}

La incorporación de los recursos digitales por parte de las organizaciones estudiadas amplifica la difusión de las acciones realizadas, usando las redes sociales para informar las 
actividades y convocar abiertamente a la colaboración con alimentos e insumos (RSO1/25/10/2020; RSO2/14/12/2021 y RSO4/06/12/2020). A lo anterior, se agrega la recepción de transferencias electrónicas de recursos económicos a las cuentas de ahorro de las organizaciones responsables por parte de donantes particulares o colectivos, destinados a la adquisición de víveres e insumos para el funcionamiento de la iniciativa. En ese mismo formato, las rendiciones de cuentas de los aportes recibidos son publicadas en las distintas redes sociales con las que cuentan las organizaciones, con el fin de transparentar los gastos realizados y responder públicamente a la confianza de las personas y organizaciones que han aportado a la actividad (RSO3/05/01/2021 y RSO4/28/11/2020). Estas acciones son avaladas con publicaciones de fotografías del proceso de preparación del menú diario y de imágenes en torno al momento de la entrega de los alimentos a las familias beneficiadas (RSO2/16/11/2020 y RSO3/05/01/2021), en un interesante ejercicio de registro orientado a la transparencia de las acciones realizadas y a la difusión del efectivo destino final de los recursos recolectados.

Desde los antecedentes revisados, puede afirmarse que a pesar de que las políticas postdictatoriales desconocieron la histórica capacidad de organización de los sectores poblacionales (Angelcos, 2016), la reedición de las ollas comunes en contextos de crisis por parte de las organizaciones sociales es una evidencia de la vigencia permanente de las prácticas de resistencia en el mundo social, las que han sido capaces de trascender la omisión normativa institucional y generar una cadena de acciones orientadas a manifestar la disconformidad social por las condiciones de funcionamiento institucional del país y que en las comunas estudiadas se han centrado principalmente en torno a las temáticas ambientales y habitacionales. Así, en la última década las prácticas de resistencia precedentes han estado asociadas a manifestaciones de rechazo frente a las presiones que ejercen las inmobiliarias sobre el suelo urbano, destinadas a imponer forzadamente su interés habitacional por sobre el destino original de remanentes, áreas verdes o parques urbanos que poseen los terrenos en disputa.

Como respuesta, las organizaciones de pobladores realizan prácticas de resistencia colectivas que han buscado la ocupación social del territorio a través de la recuperación ambiental de espacios comunitarios degradados, para visibilizar el interés social frente a la amenaza del interés inmobiliario. Asimismo, en forma paralela se han observado ocupaciones de hecho de predios urbanos por parte de familias que carecen de soluciones habitacionales, reeditando las acciones de "tomas de terrenos" que caracterizaron anteriores etapas históricas de crisis sociales y económicas en el país, compartiendo actualmente esta acción con familias migrantes que buscan con urgencia mecanismos que les permitan establecerse en forma más estable, aun cuando sea en condiciones de precariedad material.

Estas acciones de resistencia resultan consistentes con los planteamientos de NúñezPuente, Fernández-Romero y Farné (2018) ya que han permitido definir en el tiempo una 
clara posición política basada en los principios y valores comunitarios compartidos por las organizaciones de pobladores, por sobre los intereses mercantiles. A partir de dichos antecedentes, la crisis social iniciada en Octubre del 2019 se enlazó centralmente con el proceso anterior, generando un importante incremento de nuevas prácticas de resistencia, al sumar renovadas manifestaciones de protesta basadas en la comunidad y expresadas en cabildos auto convocados, actos de homenaje, toque de cacerolas, marchas, bloqueos de calles, murales, lienzos e instauración de las primeras ollas comunes en algunos de los sectores de las comunas estudiadas.

La restauración de la solución organizada para la alimentación comunitaria fue visibilizada inicialmente como una acción simbólica, transformándose posteriormente en una práctica recurrente, en la medida que las condiciones económicas de las familias comenzaron a presentar un rápido deterioro como resultado del impacto de la emergencia sanitaria iniciada en marzo del año 2020. En efecto, las restricciones aplicadas en el funcionamiento de las actividades económicas y las medidas de confinamiento de la población han afectado directamente las oportunidades de empleo de la población de bajos ingresos, especialmente entre quienes realizan trabajos ocasionales, servicio doméstico o poseen pequeños emprendimientos ligados al comercio o a los servicios personales. Esta resistencia no solo se expresa en la organización social por sí misma, sino que se enlaza con las condiciones de tránsito cotidiano a la olla común, en que sortear los escollos de las restricciones de movilidad, representa enfrentarse directamente a los riesgos sanitarios del contagio y sus consecuencias. Con ello, la evidencia de la alimentación como una necesidad básica insustituible se sitúa por sobre la protección de la propia salud, develando que una práctica de resistencia puede superar los límites críticos del riesgo personal y la sanción formal.

Coincidente con Collignon-Goribar (2010) la organización comunitaria en torno a la alimentación representa una práctica de resistencia que desafía abiertamente los referentes tradicionales. Tomando como base dicho planteamiento y analizando la información recopilada, es posible constatar que las organizaciones sociales estudiadas expresan sus posiciones antagónicas a través de tres dimensiones. La primera dimensión es la práctica de resistencia como denuncia, dado que la realización de la actividad llama la atención en forma pública sobre la precaria situación económica de las familias que acuden a la actividad y que se encuentran impedidas de satisfacer sus necesidades de alimentación por medio de sus propios recursos. Esta pública llamada de atención pone el acento sobre la incapacidad demostrada por las acciones gubernamentales para garantizar apoyos materiales o financieros permanentes que permitan cubrir adecuadamente las necesidades básicas de la población afectada por el actual contexto de crisis sanitaria. Para ello, los mecanismos de captura de la atención se expresan a través de modalidades de interacción directa en torno a la información que circula a nivel comunitario; y de interacción virtual de la que circula en redes sociales, en donde se difunden en el marco de un amplio auditorio 
capaz de otorgar una mayor resonancia a las actividades, al desanclarla del relato construido en el territorio y proyectándola a incontables nuevas audiencias. A lo anterior se suma el hecho de que el actual funcionamiento de las ollas comunes ha sido cubierto por medios de prensa, en atención a que no representan una práctica en condiciones de clandestinidad o afecta a censura, como sucedió en los pasados tiempos dictatoriales, por lo que la difusión de sus actividades se realiza en forma abierta por los diferentes medios de comunicación social disponibles, colaborando con la expresión masiva de la resistencia como acción de denuncia.

La segunda dimensión refleja la práctica de resistencia como un abierto desafío al modelo económico vigente, en atención a que la búsqueda de soluciones colectivas es opuesta a la concepción del modelo de desarrollo imperante que apuesta a las lógicas del individualismo y la competitividad para alcanzar la satisfacción de necesidades personales y familiares. Las prácticas de resistencia realizadas en torno a la necesidad de alimentación que se han analizado constituyen respuestas organizadas, en donde la entrega de raciones preparadas en forma colectiva fomentan valores y acciones asociadas a la solidaridad, la autogestión, el trabajo mutuo y la colaboración entre pares, con el fin de evitar instalar dinámicas predominantemente asistenciales como único patrón de respuesta a las necesidades y problemas comunes. La tercera dimensión sitúa la solución colectiva de la alimentación como un acto de dignidad, capaz de acoger a la diversidad de los sujetos sociales presentes en el territorio sin discriminaciones originadas en mediciones de materialidades económicas o focalizaciones instrumentales, tal como ocurre con las políticas sociales vigentes. Por el contrario, la expresión de solicitud del beneficio es la principal evidencia de una necesidad que requiere ser acogida y resuelta en el marco de los esfuerzos colectivos realizados.

Estas tres dimensiones se encuentran en la base del reposicionamiento de las organizaciones pobladoras como agentes sociales y políticos activos, expresando en su discurso y su acción una perspectiva abiertamente cuestionadora de la concepción de Estado subsidiario vigente, cuyos mecanismos de apoyo entregados en contexto de pandemia se han implementado invariablemente desde concepciones de focalización, obligando nuevamente a vastos sectores sociales a competir entre ellos para acceder a recursos escasos y fuertemente condicionados por requisitos predefinidos desde lógicas tecnocráticas, por sobre consideraciones sanitarias y sociales.

Junto con lo anterior, debe mencionarse como antecedentes de contexto que las actuales organizaciones sociales de pobladores se diferencian de las experiencias históricas documentadas, a partir de los niveles de escolaridad formal que poseen sus dirigentes y gran parte de su membresía. Según los antecedentes aportados por Hardy (1986), la escolaridad formal de las personas participantes de ollas comunes en la década de 1980 alcanzaba en promedio la enseñanza primaria incompleta. Actualmente, en las experiencias 
estudiadas se constata que los promedios de años de estudio de las personas que participan en las organizaciones sociales se elevan a enseñanza secundaria completa y, en ocasiones, alcanzan a la formación superior, en sus niveles técnico y profesional. Este antecedente permite profundizar los fundamentos de las prácticas de resistencia, confrontar las respuestas oficiales recibidas desde argumentos y contrargumentos que apuestan por el debate y la confrontación abierta cuando se encuentra en juego la verificación de la información recibida, la asignación de recursos y la adecuada justificación de las decisiones públicas en los ámbitos económico, social y sanitario. Con ello, suman al discurso histórico de reivindicación social y de satisfacción de necesidades básicas que había caracterizado hasta ahora a las organizaciones sociales de pobladores, el reconocimiento a la vulneración de derechos económicos y sociales que se conjuga en la actual emergencia sanitaria y la incapacidad de las elites políticas para ofrecer alternativas pertinentes y oportunas a los sectores que están siendo afectados en forma directa por la pandemia.

Asimismo, las argumentaciones críticas de la población en la crisis social del año 2019 que precedió a la actual emergencia sanitaria, pusieron el acento en las condiciones de funcionamiento institucional del país, fundamentos que aportan mayor trascendencia a la narrativa social vigente y aportan nuevos alcances a la implementación de las prácticas de resistencia actualmente en curso. Amplifican estas condiciones el manejo intermedio y avanzado que poseen las nuevas generaciones de las tecnologías de información y comunicación, lo que hace posible el acceso instantáneo a prensa alternativa, difusión de prácticas sociales de denuncia, acceso a información sensible o convocatorias a protesta social, las que dinamizan los repertorios de los discursos sociales y de las prácticas de resistencia, a una velocidad de configuración que supera con creces la capacidad de respuesta oficial de las autoridades formales y de los organismos públicos.

Finalmente, puede plantearse que los resultados obtenidos coinciden con los planteamientos de Alves da Silva y Barreta-Silva (2020) y Jarpa-Arriagada (2020), dado que las ollas comunes pueden ser reconocidas como prácticas de resistencia que cuestionan directamente al modelo de desarrollo neoliberal, el que se ha demostrado incapaz de enfrentar las consecuencias económicas y sociales derivadas de la pandemia. Como contraparte de esta evidente incapacidad, las ollas comunes han acogido la atención de la necesidad de alimentación de amplios sectores de la población, como una estrategia colectiva de denuncia social que desafía a las normativas vigentes y se preocupa por cautelar la dignidad de sus participantes. Se transforman así en iniciativas que buscan reducir la subordinación y dependencia de la población, respecto de las acciones asistenciales e individuales impulsadas desde una subsidiariedad pública que se ha visto superada en su obligación de resguardo de las necesidades básicas, en el contexto de la emergencia sanitaria en curso. 


\section{Conclusiones}

En un creciente contexto de prácticas de resistencia realizadas por las organizaciones sociales chilenas en la última década, las consecuencias económicas y sociales de la emergencia sanitaria por COVID-19 han profundizado el malestar social de la población, por lo que su contenido ha sido integrado a las dinámicas de reivindicación política que representan las iniciativas analizadas. En efecto, las ollas comunes han acogido las diversas situaciones sociales de precariedad económica que afectan directamente a las familias pobladoras y su cada vez más difícil búsqueda de ingresos en condiciones de confinamiento, aportando una respuesta operativa a las necesidades básicas insatisfechas y realizando a la vez una abierta crítica social a las insuficientes acciones del Estado para enfrentar la pandemia y sus restricciones de movilidad. En la presente investigación, las organizaciones sociales de pobladores constituyen una red territorial de prácticas de resistencia ligadas a la búsqueda de estrategias colectivas de sobrevivencia frente a la necesidad básica de alimentación que afecta a la población de menores ingresos. Dichas prácticas han redefinido los contextos comunitarios desde territorios pasivos afectados por la pobreza hacia territorios activos de autoafirmación y de resistencia. La redefinición transformadora derivada de las redes colaborativas presenciales y virtuales, permite la entrega de apoyo entre pares y visibiliza importantes consecuencias políticas, al revelar las tramas del tejido social que enfrenta la pandemia con un nivel de precariedad y carencia de tal profundidad, que los recursos públicos y las acciones institucionales de beneficencia no logran aportar coberturas mínimas de satisfacción permanente a sus requerimientos personales y familiares.

La progresiva tensión social que se produce entre las prácticas de resistencia que realizan las organizaciones sociales de pobladores y las medidas gubernamentales disponibles para enfrentar la crisis sanitaria devela las posiciones antagónicas en juego, siendo sus puntos críticos la adecuada cobertura de los beneficios sociales y la efectiva disponibilidad de recursos con fines de atención social. En el marco de un modelo económico de libre mercado y de un rol estatal subsidiario, los efectos negativos de la tensión recaen sobre la población, amplificando las consecuencias de las crisis social y sanitaria sucedidas en los últimos dos años a escala nacional y mundial, permitiendo declarar el resultado de las posiciones antagónicas como una asignatura pendiente, que sigue acumulando manifestaciones de malestar social. Como corolario, puede afirmarse que las ollas comunes constituyen una práctica social que actualiza las memorias de resistencia de las organizaciones sociales de pobladores, a través de estrategias históricas de sobrevivencia colectivas que les permiten enfrentar sus carencias en forma dinámica. En ellas, asumen una posición política explícita basada en la comunidad e implementan acciones transformadoras que valoran positivamente la acción colectiva por sobre la acción individual, develando las situaciones de necesidad y carencia que son invisibilizadas desde los criterios tecnocráticos de asignación de recursos estatales. Si bien las prácticas de 
Castañeda-Meneses

resistencia no pueden detener por sí mismas los efectos de la pandemia sanitaria, los resultados de la investigación indican que constituyen prácticas propositivas que se reencuentran con la tradición organizacional de protección de los Derechos Humanos de la población, enfrentando los efectos sociales de la emergencia sanitaria a través de respuestas que ofrecen una protección social solidaria y desafían abiertamente a los estrechos criterios de subsidiariedad y focalización declarados en las políticas sociales neoliberales del Chile post dictatorial.

\section{Referencias bibliográficas}

Alves da Silva, R., y Barreto-Silva, R. (2020). Estrategias económicas alternativas en América Latina: ¿prácticas de resistencia instrumentos de transformación? En E. Pérez y A. Gonzales (Eds.) Políticas Públicas. Estrategias económico alternativas y derechos económicos de las mujeres (pp. 177-195). País Vasco: Hegoa Instituto de Estudios sobre Desarrollo y Cooperación Internacional. Universidad Nacional Mayor de San Marcos (UNMSM). Recuperado de https:/ / publicaciones.hegoa.ehu.eus/uploads/pdfs/461/_Politicas_Publicas.pdf?16 01979247.

Angelcos, N. (2016). Movimiento de pobladores. Lucha social y política en el Chile contemporáneo. Educação em Perspectiva, 7(2) 324-345. Recuperado de https://coes.cl/publicaciones/movimiento-de-pobladores-lucha-social-y-politicaen-el-chile-contemporaneo/.

Barreneche, C., Lombana-Bermúdez, A., y Ramos-Martín, J. (2021). Datificación en contextos de corrupción: imaginarios sociotécnicos y prácticas de resistencia frente a sistemas antipobreza en Colombia. Palabra Clave, 24(3), e2434. doi: 10.5294/ pacla.2021.24.3.4

Berlagoscky, F., Infante, A., y Ferrari, I de. (2018). La participación social en dictadura: protagonismo popular. En Cuadernos Médico Sociales 58(3) 29-32. Recuperado de https:/ / www.researchgate.net/profile/Ximena-

Barros/publication/332257932_Unveiling_the_social_value_of_primary_care_in_Ch ile/links/5ca9fb19a6fdcca26d053de1/Unveiling-the-social-value-of-primary-care-inChile.pdf\#page $=31$.

Campos y Covarrubias, G., y Lule-Martínez, N. (2012). La observación, un método para el estudio de la realidad. Revista Xihmai 7(13), 45-60. Recuperado de https:/ / dialnet.unirioja.es/servlet/articulo?codigo=3979972.

Collignon-Goribar, M. M. (2011). Discursos sociales sobre la sexualidad: narrativas sobre la diversidad sexual y prácticas de resistencia. Comunicación y sociedad, (16), 133-160. Recuperado de http://www.scielo.org.mx/pdf/comso/n16/n16a6.pdf.

Ffrench-Davis, R., y Stallings, B. (Eds.). (2001). Reformas, crecimiento politicas sociales en Chile desde 1973. Santiago de Chile: CEPAL/LOM. 
Castañeda-Meneses

Gallardo, B. (1985a). El redescubrimiento del carácter social del problema del hambre. Las ollas comunes. Santiago, Chile: FLACSO. Recuperado de http:/ / flacsochile.org/biblioteca/pub/memoria/1985/000931.pdf.

Gallardo, B. (1985b). Las ollas comunes de La Florida como experiencia de desarrollo de la organización popular. Santiago, Chile: FLACSO. Recuperado de http:/ / flacsochile.org/biblioteca/pub/memoria/1985/000932.pdf.

Gobierno de Chile. (2020). Plan de reactivación económica. Recuperado de https://www.gob.cl/juntosporchile/reactivacioneconomica/.

Guerrero-Jiménez, B., y Pérez-Mora, A. (2020). Estallido social y pandemia: de los cabildos a las ollas comunes. El caso del Norte Grande de Chile. Espacio Abierto. Cuaderno Venezolano de Sociología, 29(4), 106-117. Recuperado de https://www.redalyc.org/jatsRepo/122/12265803006/12265803006.pdf.

Hardy, C. (1986). Hambre + Dignidad = Ollas Comunes. Santiago, Chile: LOM/EUROSOCIAL.

Herrera, J. (2018). El nuevo movimiento de pobladores en Chile: el movimiento social desplazado. Polis. Revista Latinoamericana, (49), 177-199. Recuperado de https://polis.ulagos.cl/index.php/polis/article/view/1332/2522.

Iglesias-Vázquez, M. (2016). La construcción teórica de los movimientos sociales en Chile: el movimiento de pobladores, entre la Sociología y la Historia Social. Revista Austral de Ciencias Sociales, (30), 145-160. Recuperado de http:/ / revistas.uach.cl/pdf/racs/n30/art07.pdf.

Instituto Nacional de Estadísticas Chile [INE]. (2017). Resultados Censo 2017. Recuperado de http:// resultados.censo2017.cl/.

Jarpa-Arriagada, C. G. (2020). Prácticas de resistencia y trabajo social comunitario: forcejeos y tensiones ante las lógicas de dominación del modelo colonial y capitalista. Eleuthera, 22(2), 309-326. doi: 10.17151/eleu.2020.22.2.18.

Jociles-Rubio, M. I. (2018). La observación participante en el estudio etnográfico de las prácticas sociales. Revista Colombiana de Antropología,54(1), 121-150. doi: $10.22380 / 2539472 X .386$

Langer, E. (2016). La construcción de confianza para el estudio de prácticas de resistencia en la escolarización de jóvenes en contextos de pobreza urbana. Revista Interamericana de Educación, Pedagogía y Estudios Culturales, 9(2), 113-137. Recuperado de https://ri.conicet.gov.ar/bitstream/handle/11336/116224/CONICET_Digital_Nro. 33d6d58c-c52f-4520-bc97-be7b79c7b6fa_A.pdf?sequence=2\&isAllowed=y.

Ministerio de Salud de Chile [MINSAL]. (2020). Plan de Acción Coronavirus COVID-19. Recuperado de https://www.minsal.cl/nuevo-coronavirus-2019-ncov/casosconfirmados-en-chile-covid-19/.

Morales-Quiroga, M. (2020). Estallido social en Chile 2019: participación, representación, confianza institucional y escándalos públicos. Análisis Político, 33(98), 3-25. doi: 10.15446/anpol.v33n98.89407. 
Castañeda-Meneses

Núñez-Puente, S., Fernández-Romero, D., y Farné, A. (2018). Comunicación, violencia de género y prácticas de resistencia: narrativas innovadoras para un cambio social. Teknocultura, 15(2), 185-192. Recuperado de http:/ / repositori.uji.es/xmlui/bitstream/handle/10234/180903/62851.pdf?sequenc $\mathrm{e}=1$ \&isAllowed $=\mathrm{y}$.

Palacios-Sepúlveda, F. (2020). La participación y rol de las mujeres de sectores populares en ollas comunes. Quito, Ecuador: Universidad de Las Américas. Facultad de Ciencias Sociales. Programa de Intervención Comunitaria. Recuperado de https://www.udla.cl/wpcontent/uploads/2020/07/6-La-participaci\%C3\%B3n-y-rol-de-las-mujeres-desectores-populares-en-ollas-comunes.pdf.

Piñeiro, D. (1985). Formas de resistencia de la agricultura familiar: el caso del noreste de Canelones. Montevideo, Uruguay: CIESU.

Ramos, C. (2020). Covid-19: la nueva enfermedad causada por un coronavirus. Salud Pública de México, 62(2), 225-227. doi: 10.21149/11276.

Razeto-Migliaro, L. (1986). Economía popular de solidaridad. Identidad y proyecto en una versión integradora. Santiago, Chile: Área Pastoral Social de la Conferencia Episcopal de Chile. Programa de Economía del Trabajo-PET.

Rivera-Aguilera, G. (2017). Los procesos de influencia global/local en políticas públicas: una propuesta metodológica. Psicoperspectivas. Individuo y Sociedad, 16(3), 111-121. doi: 10.5027/ psicoperspectivas-vol16-issue3-fulltext-1092.

Valdés, T. (1986). El movimiento poblacional: la recomposición de las solidaridades sociales. Santiago, Chile: FLACSO. Recuperado de http://www.bibliotecanacionaldigital.gob.cl/visor/BND:9579.

Valdés, T., Weinstein, M., y Malinarich, A. (1988). Las Coordinadoras de Organizaciones Populares. Cinco experiencias. Santiago, Chile: FLACSO. Recuperado de http:/ / flacsochile.org/biblioteca/pub/memoria/1988/000184.pdf.

Valenzuela-Contreras, C. (2021). Mujeres, diversidades sexuales y de género ante la crisis por COVID: Aportes decoloniales y feministas. En C. A. Caro-Zuñiga (Comp.), Covid19, Feminismo Decolonial y Revueltas Populares. Dossier $N^{\circ} 7$ del programa de Estudios Comunitarios Latinoamericanos de la Universidad de Chile: COVID-19, feminismo decolonial y revueltas populares (pp. 23-30). Chile: Universidad de Chile. Facultad de Ciencias Sociales. Programa de Estudios Comunitarios Latinoamericanos. doi: 10.34720/c5e9$4 z 65$.

Valles, M. (2000). Técnicas cualitativas de investigación social. Reflexión metodológica y práctica profesional. Madrid, España: Síntesis. 


\section{OTROS ARTÍCULOS DE PROSPECTIVA No. 33}

\section{EDITORIAL}

El Paro Nacional en Colombia 2021: explosión social entre dinámicas estructurales y de coyuntura. Relevancia de la acción política y del diálogo en su desarrollo y transformación Adolfo Adrián Álvarez-Rodríguez

\section{ARTÍCULOS}

Organizaciones sociales de pobladores y prácticas de resistencia en contexto de pandemia COVID-19 en Chile

Patricia Castañeda-Meneses

Entre el aislamiento y las brechas digitales: sistematización de experiencia de acompañamiento socioemocional en personas mayores de Temuco, Chile, en tiempos de COVID-19

G. Bernarda Aedo-Neira

Percepción de apoyo social y calidad de vida: la visión de personas mayores chilenas en el contexto de pandemia durante el 2020

Claudia Elena Quiroga-Sanzana

Gabriela Rocío Parra-Monje

Camila Julia Moyano-Sepúlveda

Marco Alejandro Díaz-Bravo

A propósito del qué, cómo y para qué investigar en el campo de la justicia juvenil: La trastienda de un proceso de investigación en Rosario, Argentina Karina De Bella

Proyección social: reflexión sobre una experiencia virtual con adolescentes en época de pandemia en Sucre y Bolivar, Colombia

Claudia Yaneth Martínez-Mina
La intervención social mediante proyectos comunitarios en modalidad virtual en Mérida, México. Una perspectiva desde el Trabajo Social Claudia Isabel Tzec-Puch Amairani Aracelly Ceh-Alvarado Yanet Guadalupe González-Canul

Resignificación de los vínculos Sociales durante la pandemia de COVID-19: narrativas de personas que convergen en Conjuntos residenciales en Bogotá, Colombia

Andrea Marcela Reyes-García

Laura Daniela Molina-Ortiz

La paradoja de la familia doblemente recluida: clínica de una pandemia en Colombia

Norman Darío Moreno-Carmona

Juan José Cleves-Valencia

Ciencias sociales, mundo y pandemia: por un futuro posible

José Miguel Segura-Gutiérrez

Lina Paola Vásquez-Ávila

¿Qué es Trabajo Social? Los primeros vínculos de los estudiantes con la profesión: experiencias de Argentina, Brasil, Paraguay y Uruguay

Silvia Orieta Rivero-Rodríguez

Leonel Del Prado

Nidia Graciela Battilana-Amarilla

Rosilaine Coradini-Guilherme

Intervención profesional de trabajadores sociales de la universidad del Quindio, Colombia. Asuntos epistemológicos, metodológicos y ético-políticos

Ana María Gil-Ríos 
Reflexiones sobre la intervención social en lo rural: experiencias en el Magdalena Medio, Colombia

Claudia Milena Quijano-Mejía

Johana Linares-García

Construcción de rutas de atención integral a la convivencia escolar en Cali, Colombia: sistematización de la experiencia

Leidy Johana Prado-Montaño

Luz Helena López-Rodríguez

Alejandra Gutiérrez-Cárdenas

Experiencia del proceso de intervención familiar de los actores participantes en la Escuela para Familias del ICBF-Jamundí, Colombia Katherin Viviana Silva-Minotta

María José Forero-Izquierdo

Jimena del Pilar Jaramillo-Jaramillo

\section{RESEÑAS DE LIBROS}

Alerta global: políticas, movimientos sociales y futuros en disputa en tiempos de pandemia Jairo Crispín

El neoliberalismo como teología política. Habermas, Foucault, Dardot, Laval y la historia del capitalismo contemporáneo

José Francisco Desentis-Torres

La Reconceptualización del Trabajo Social en Colombia: Análisis histórico-crítico de las décadas de 1960-1970

Juan Pablo Sierra-Tapiro

ARTISTA INVITADO

Juan Camilo González

Prospectiva

\section{PROSPECTIVA}

Revista de Trabajo Social e Intervención Social

No. 33 • ene.-jun. 2022

e-ISSN: 2389-993X • Universidad del Valle 